\title{
Management of Post-LASIK Dry Eye with Intense Pulsed Light in Combination with $0.1 \%$ Sodium Hyaluronate and Heated Eye Mask
}

\author{
Yi Wu • Ling Xu • Yilin Song • Qing Zhang · Guanghao Qin • \\ Lanting Yang $\cdot$ Jinfei Ma $\cdot$ Christoph Palme $\cdot$ Jonathan E. Moore • \\ Emmanuel Eric Pazo (D) - Wei He
}

Received: September 7, 2021 / Accepted: October 25, 2021 / Published online: November 6, 2021

(c) The Author(s) 2021

\begin{abstract}
Introduction: Laser in situ keratomelieusis (LASIK) is one of the most frequently performed refractive treatments. Dry eye (DE) is common in patients after LASIK and can be bothersome postoperatively. Therapies such as intense pulsed light (IPL), sodium hyaluronate (SH) and heated eye mask (HEM) have been reported to improve signs and symptoms of DE .
\end{abstract}

Y. Wu · L. Xu · Y. Song · Q. Zhang · G. Qin ·

L. Yang · E. E. Pazo $(\bowtie) \cdot$ W. He $(\bowtie)$

Department of Ophthalmology, He Eye Specialist

Hospital, No. 128 North Huanghe Street, Shenyang

1100 34, China

e-mail: ericpazo@outlook.com

W. He

e-mail: hsyk2017@163.com

Y. Wu · Y. Song · Q. Zhang · G. Qin · L. Yang The Second Affiliated Hospital of Dalian Medical University, Dalian, China

J. Ma

Zhejiang University School of Medicine First

Affiliated Hospital, Breast Cancer, Hangzhou,

Zhejiang, China

C. Palme

Department of Ophthalmology and Optometry,

Medical University of Innsbruck, Innsbruck, Austria

J. E. Moore

Cathedral Eye Clinic, 89-91 Academy Street, Belfast, UK
Aim: The purpose of this prospective study was to evaluate and compare the effects of IPL and $0.1 \%$ SH (IPL group, 50 eyes) and IPL in combination with $0.1 \%$ SH and HEM (IPL + group, 50 eyes) in participants with persistent post-LASIK DE.

Methods: The final analysis included 100 patients (100 eyes) who had LASIK for myopic correction and had been experiencing moderate to severe DE following LASIK for over a year. Participants were randomly assigned to either the IPL group (2 IPL sessions) or IPL + group (2 IPL sessions and daily HEM for 4 weeks), and both groups continued the use of daily $0.1 \% \mathrm{SH}$ (HYLO-COMOD $\left.{ }^{\circledR}\right)$ preservative-free eye drops. Non-invasive tear break-up time (NITBUT), tear film lipid layer (TFLL), lower tear meniscus height (LTMH), meibomian gland quality (MGQ), meibomian gland expressibility (MGEx), corneal fluorescein staining (CFS), ocular surface disease index (OSDI) and artificial tear usage (ATU) survey were assessed at baseline (BL) and follow-up at 2 (F1) and 4 weeks (F2).

Results: Following the treatment protocol, all dry eye (DE) parameters assessed in this study improved significantly $(P<0.05)$ in both groups at F2 compared with their respective BL measurements. Inter-group comparison at F2 found significant differences in their NITBUT (IPL: $\quad 6.06 \pm 0.59$ vs. $\quad$ IPL $+: \quad 6.67 \pm 0.86$, $P<0.001$ ), TFLL (IPL: $1.90 \pm 0.65$ vs. IPL + : $1.60 \pm 0.64, \quad P=0.021), \quad$ LTMH $\quad$ (IPL: $0.186 \pm 0.053$ vs. $0.204 \pm 0.034, \quad P=0.003)$, MGQ (IPL: $1.48 \pm 0.54$ vs. IPL $+: 1.26 \pm 0.56$, 
$P=0.026$ ), MGEx (IPL: $1.62 \pm 0.53$ vs. IPL + : $1.44 \pm 0.50, \quad P=0.038$ ) and OSDI (IPL: $32.54 \pm 6.85 \quad$ vs. $\quad$ IPL $+: \quad 29.76 \pm 4.74$, $P=0.001$ ), while CFS score (IPL: $4.02 \pm 0.65$ vs. IPL $+: 3.96 \pm 0.73, P=0.652$ ) and ATU (IPL: $1.88 \pm 0.63$ vs. IPL $+: 1.72 \pm 0.50, P=0.159)$ showed no significant difference.

Conclusion: Post-LASIK DE signs and symptoms can be improved by combining therapies such as IPL, HEM and $0.1 \%$ SH. Increased TFLL due to a combination of IPL, $0.1 \%$ SH and HEM had a greater positive impact on the subjective and objective DE measurements in participants with persistent post-LASIK DE.

Keywords: Heated eye mask; IPL; Meibomian glands; Post-LASIK dry eye; Tear film lipid layer

\section{Key Summary Points}

\section{Why carry out this study?}

Dry eye is common in patients after LASIK and can be bothersome postoperatively. Heated eye mask, intense pulsed light and $0.1 \%$ sodium hyaluronate have been found to be beneficial in reducing signs and symptoms of dry eye.

This study aimed to assess whether the combination of intense pulsed light with heated eye mask and $0.01 \%$ sodium hyaluronate (study group) was more effective than intense pulsed light and $0.01 \%$ sodium hyaluronate (active control group) in participants with post-LASIK dry eye.

\section{What was learned from the study?}

This is the first study to our knowledge to assess and conclude that combining intense pulsed light with heated eye mask and $0.01 \%$ sodium hyaluronate is an effective treatment to ameliorate signs and symptoms of post-LASIK dry eye.

The combined treatment was found to be more effective than intense pulsed light and $0.01 \%$ sodium hyaluronate.
Increase in tear film lipid layer may have been the contributing factor that led to a greater positive impact on the subjective and objective dry eye measurements in participants undergoing combined treatment.

\section{INTRODUCTION}

Refractive interventions such as cataract surgery, photorefractive keratectomy (PRK) [1], small incision lenticule extraction (SMILE) [2] and femtosecond laser-assisted in situ keratomileusis (LASIK) operations have been documented to negatively alter the ocular surface environment and result in tear film instability [3]. According to the DEWS convention, dry eye disease (DED) is defined as "a multifactorial disease of the tears and ocular surface that results in symptoms of discomfort, visual disturbance, and tear film instability with potential damage to the ocular surface. It is accompanied by increased osmolarity of the tear film and inflammation of the ocular surface" [4]. Dry eye (DE) symptoms in patients can result in discomfort, photophobia and vision loss with varying ranges of severity [4], and studies have found that although DE symptoms following LASIK are common and transient in occurrence, incidences of refractory post-LASIK $\mathrm{DE}$ are few $[5,6]$. Although there are various mechanisms regarding the pathophysiology of post-LASIK DE, the primary cause is considered to be iatrogenic corneal nerve severing during the surgery as the dense sub-basal nerve plexus and stromal corneal nerves are severed during the anterior stromal flap creation process by the excimer laser [6, 7]. Additionally, decrease in conjunctival goblet cells has also been proposed to exacerbate post-LASIK DE [8, 9] . Similar to DED [10], the post-LASIK DE state has been hypothesized to be related to inflammation $[11,12]$. In addition, changes to the corneal shape due to LASIK have also been reported to negatively alter the interaction among the eyelids, ocular surface and tear film distribution 
[13]. As mentioned above, corneal nerve severing has been reported to decrease the corneal sensitivity and corneal blinking reflex, which in turn decrease meibomian gland secretion because if is controlled by blinking [14]. Reduced blink rate can lead to increased tear evaporation, since incomplete blinking induces meibomian gland dysfunction (MGD) and delayed tear clearance $[15,16]$.

Topical sodium hyaluronate (SH) ophthalmic drops (preferably preservative free) have emerged as a first-line treatment of choice for DED. They owe their efficacy to hyaluronic acid (HY), which is a naturally occurring linear biopolymer consisting of repeating disaccharide units of N-acetyl-D-glucosamine and sodium-Dglucoronate. HY is widely used today and has been shown to result in both subjective and objective improvement in DED subjects [17]. Intense pulsed light (IPL) is widely used to treat dermatological conditions [18, 19], and its noncoherent polychromatic light source with a wide wavelength range of $500-1200 \mathrm{~nm}$ has been reported to stimulate facial sebaceous glands [20]. The photothermal effect of IPL is postulated to relieve inflammation by removing aberrant surface microvasculature and enhances meibomian gland function [21, 22]. Furthermore, an increase in fibroblast proliferation, collagen formation and local blood flow has been associated with the application of IPL on the skin $[23,24]$. Several studies have documented the benefits of IPL in alleviating signs and symptoms of DED on the periocular skin $[25,26]$. Eyelid warming devices such as heated eye masks (HEMs) boost meibum secretions by liquefying meibum release into the tear film and relieving symptoms associated with dry eye [27]. Since the expression of meibum is evoked by eyelid blinking, MGD can be provoked by a reduction of blinks. Since corneal denervation by LASIK disrupts the reflex controlling the lacrimal gland and blinking, the "circle" of events triggered by the surgery decreases corneal sensitivity and blinking frequency, leading to sub-optimal meibomian gland secretion and increased tear evaporation. While the pathophysiology of transient and persistent postLASIK DE is complex and multifactorial [6], clinical research findings on the benefits of using artificial tears containing $\mathrm{SH}$ have been widely reported and typically used as the firstline treatment for DED and post-LASIK DE [28]. However, in some instances, failure of artificial tears in treating DE has been reported to occur [29]. Therefore the purpose of this study was to assess the management of post-LASIK DE by combining therapies such as IPL [30], 0.1\% SH and HEM.

\section{METHODS}

\section{Study Design and Participants}

This prospective study was carried out in the refractive treatment center of Shenyang He Eye Specialist Hospital, which recruited 50 participants clinically diagnosed with moderate to severe post-LASIK DE for over a year. All participants were diagnosed according to the Japanese DE diagnostic criteria [31]: (1) presence of dry eye symptomatology; (2) presence of either qualitative or quantitative disturbance of the tear film (Schirmer test $<5 \mathrm{~mm}$ or TBUT $<5 \mathrm{~s}$ ); (3) presence of conjunctivocorneal epithelial damage (fluorescein staining score $>3$ points, rose bengal staining score $>3$ points or lissamine green staining score $>3$ points) and had no effective recovery using traditional DE treatment. The study was conducted in compliance with the Institutional Review Board of He Eye Specialist Hospital, Shenyang, China (approval number: IRB2019.K002.01), and in accordance with the tenets of the Declaration of Helsinki. Written informed consent was obtained from the patients for publication of this study and any accompanying images. This manuscript includes no identifiable patient information.

\section{Inclusion and Exclusion Criteria}

Patients were selected on the basis of the following criteria: (1) able to provide written and signed informed consent; (2) had undergone previous LASIK surgery; (3) experiencing at least one of the following symptoms: dryness, burning, irritation, grittiness, foreign body sensation 
or fluctuating vision; (4) NITBUT $\leq 5 \mathrm{~s}$; (5) Ocular Surface Disease Index (OSDI) (Allergan, Inc., Irvine, CA) score $\geq 13$ [32]; (6) MGQ $\geq 4$ or MGEx $\geq 1$; (7) persistent symptoms of DE for 1 year of more following LASIK surgery. Participants with decreased best corrected visual acuity (BCVA) $(<20 / 20)$, active infection, eye lid disorders and any other systemic diseases affecting blinking or the ocular surface, eyelid surgeries and hereditary corneal diseases were excluded form enrolling in this study. Participants were randomly assigned to the IPL or IPL + group. Participants in the IPL group received IPL treatment only, while participants in the IPL + group received IPL along with daily disposable HEM for the entirety of the study (Fig. 1).

\section{Treatment}

The M22 IPL system (Lumenis Ltd., Yokneam, Israel) was used, which has a xenon lamp that emits IPL at $515-1200 \mathrm{~nm}$ on the cutaneous facial sebaceous glands. A 560-nm filter was adjusted to the appropriate setting (range of $11-14 \mathrm{~J} / \mathrm{cm}^{2}$ ). IPL treatment intensity was chosen based on the Fitzpatrick scale as follows: Fitzpatrick scale I, II and III, $10-15 \mathrm{~J} / \mathrm{cm}^{2}$, with a $570-n m$ filter. A total of two IPL treatment sessions were administered, once each at a 2 -week interval, to all participants (IPL and IPL + ). Bilateral IPL treatment was administered at day 0 and day 14. All participants in both groups used topical ophthalmic $0.1 \% \mathrm{SH}$ daily. The HEM (Fig. 2) used for this study was a medically certified air-activated disposable eye mask (Ocuface Medical Co., Ltd., Guangzhou, China) [33]. Participants in the IPL + group used HEM for $10 \mathrm{~min}$ once daily for 28 days along with two sessions of IPL, 2 weeks apart.

\section{Subjective and Objective Tests}

Non-invasive tear break-up time (NITBUT) and lower tear meniscus height (LTMH) were assessed using the Keratograph $5 \mathrm{M}$ (Oculus, Germany) [34]. Interferometry tear film lipid layer
(TFLL) quality was measured using DR-1 (Kowa, Nagoya, Japan) [35], and Kojima et al.'s methods were followed while assessing corneal fluorescein staining (CFS) examinations. Meibomian gland quality (MGQ) and expressibility (MGEx) were quantified as mentioned in Yang et al.[36] DED symptoms were assessed by administrating the Chinese web version of the ocular surface disease index (OSDI) questionnaire [37]. Online survey of participants (How often do you use preservative-free artificial tears at the end of the day?) was conducted using a smartphone messaging application to identify the frequency with which the participants in this research utilized artificial tears (e.g., once a day, twice a day and so on). Subjective (Y.W.) and objective (Z.Q.) assessments were performed by different trained clinical examiners.

\section{Statistical Analysis}

Statistical Analysis in Social Sciences (SPSS) for MacOS software was used to analyze the data (version 25, IBM Corp.). Data were collected from both eyes of those who received IPL and IPL + treatment at baseline (BL), first follow-up at day 14 (F1) and second follow-up at day 28 (F2). Repeated measures analysis allowed for comparisons across time periods, while paired analyses allowed for comparisons of pre- and post-treatment data at specific time periods (F1 and F2). The Kolmogorov-Smirnoff test was used to determine the normality of variables. Analysis of variance was used to analyze ordinal variables (e.g., TFLL) and those having nonnormal distributions (ANOVA). The primary outcome measures for this study were NITBUT, LTMH, TFLL and OSDI scores before and after treatment. We performed a statistical power analysis for both the OSDI score and NITBUT at day 28 visit in the IPL and IPL + groups. The power $(1-\beta)$ was $>0.90$ at the level of $\alpha=0.025$, and the sample size was sufficient. $[38,39]$ 


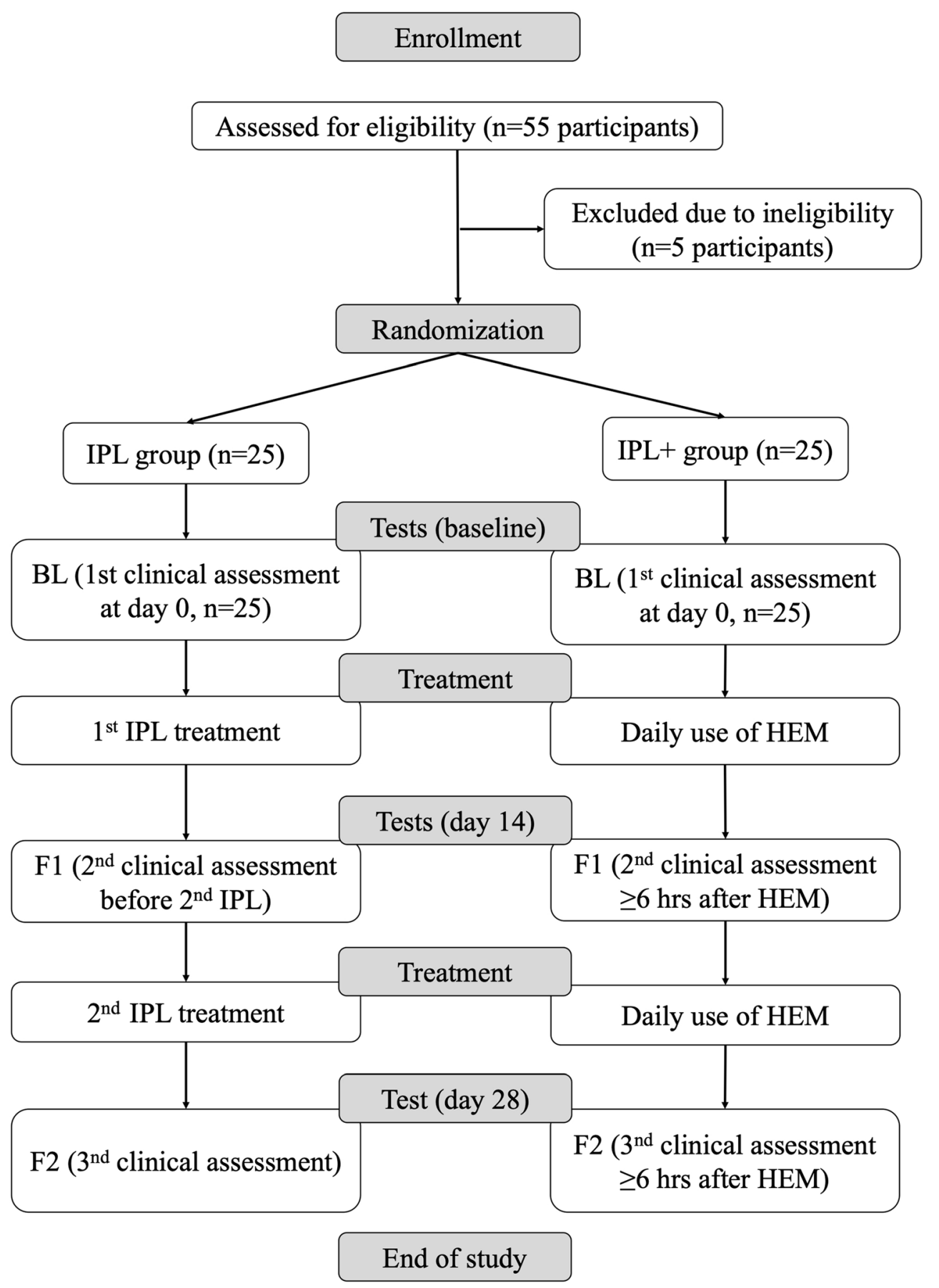

Fig. 1 Study flowchart 


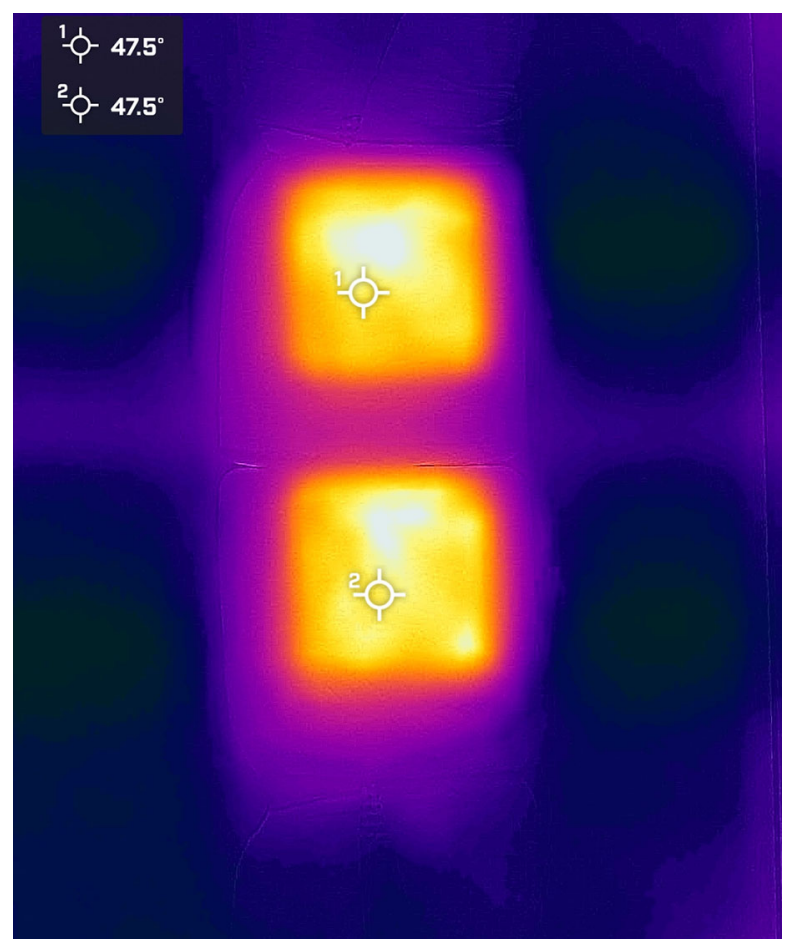

Fig. 2 Infrared image of an active, air-activated disposable heat eye mask

\section{RESULTS}

\section{Demographics}

Table 1 shows the demographic information of 100 participants (100 eyes) randomly enrolled in the IPL group and IPL + group. The mean age of participants in the IPL group and IPL + group was $31.50 \pm 4.41$ and $31.36 \pm 4.42$ years, respectively, with $37 \%$ females in both groups. There was no statistically significant difference in age $(P=0.707)$, period of post-LASIK dry eye diagnosis $(P=0.890)$ and use of artificial tears $(P=0.659)$ at baseline. Best corrected visual acuity was stable at all assessment time points (Table 2).

\section{NITBUT}

Figure 3 depicts non-invasive tear break-up time assessed at baseline, first and second follow-ups. At baseline, mean NITBUT measurements for IPL $\quad(4.03 \pm 0.59)$ and IPL $+(3.99 \pm 0.64)$ groups showed no significant difference $(P=0.304)$. However, at first follow-up $(5.00 \pm 0.66$ vs. $5.87 \pm 0.65, P<0.001)$ and second follow-up $(6.06 \pm 0.59$ vs. $6.67 \pm 0.86$, $P<0.001)$, significant differences were observed between the groups. Furthermore, $\Delta$ change in NITBUT was found to be higher in the IPL + group (2.7) than in the IPL group (2.0) (Table 2).

\section{TFLL}

Figure 4 illustrates mean changes in primary outcome measure TFLL scores at baseline and subsequent follow-up in both groups. At baseline, both groups had statistically non-significant differences between them $(P=0.098)$. During the first follow-up assessment, both groups showed statistically significant improvements compared to their respective

Table 1 Demographic information

\begin{tabular}{|c|c|c|c|c|}
\hline Parameters & IPL & $\mathrm{IPL}+$ & $F$ value & $P$ value \\
\hline No. of participants (no. of eyes) & 50 eyes & 50 eyes & - & - \\
\hline Age (mean $\pm S D)$, years & $31.50 \pm 4.41$ & $31.36 \pm 4.42$ & 0.143 & 0.707 \\
\hline Gender, female (\%) & $19(37 \%)$ & $19(37 \%)$ & - & - \\
\hline No. of months diagnosed with dry eye (mean \pm SD), months & $26.5 \pm 9.5$ & $26.6 \pm 9.6$ & 0.019 & 0.89 \\
\hline ATU (mean \pm SD) times/day & $4.46 \pm 0.84$ & $4.48 \pm 0.84$ & 0.197 & 0.659 \\
\hline
\end{tabular}

$I P L$ intense pulsed light; IPL + intense pulsed light combined with heated eye mask; $A T U$ artificial tears usage (frequency of drops per day)

${ }^{*}$ Statistically significant difference 
Table 2 Comparative clinical dry eye assessments at different time points between groups

\begin{tabular}{|c|c|c|c|c|c|c|c|c|}
\hline Parameters & Baseline (BL) & $\begin{array}{l}\text { Follow-up } \\
\text { (FU1) }\end{array}$ & $\begin{array}{l}P \text { value } \\
\text { (BL vs. } \\
\text { FU1) }\end{array}$ & $\begin{array}{l}F \text { value } \\
\text { (BL vs. } \\
\text { FU1) }\end{array}$ & $\begin{array}{l}\text { Follow-up } \\
\text { (FU2) }\end{array}$ & $\begin{array}{l}\Delta \\
\text { change } \\
\text { (BL- } \\
\text { FU2) }\end{array}$ & $\begin{array}{l}P \text { value } \\
\text { (BL vs. } \\
\text { FU2) }\end{array}$ & $\begin{array}{l}F \text { value } \\
\text { (BL vs. } \\
\text { FU2) }\end{array}$ \\
\hline $\begin{array}{l}\text { NITBUT } \\
\text { (IPL) }\end{array}$ & $4.03 \pm 0.59$ & $5.00 \pm 0.66$ & $<0.001^{*}$ & 302.524 & $6.06 \pm 0.59$ & 2.0 & $<0.001^{*}$ & 1304.731 \\
\hline $\begin{array}{l}\text { NITBUT } \\
(\mathrm{IPL}+)\end{array}$ & $3.99 \pm 0.64$ & $5.87 \pm 0.65$ & $<0.001^{*}$ & 5931.014 & $6.67 \pm 0.86$ & 2.7 & $<0.001^{*}$ & 542.957 \\
\hline$P$ value & 0.304 & $<0.001^{*}$ & - & - & $<0.001^{*}$ & - & - & - \\
\hline$F$ value & 0.584 & 179.16 & - & - & 21.179 & - & - & - \\
\hline $\begin{array}{l}\text { TFLL } \\
\text { (IPL) }\end{array}$ & $3.36 \pm 0.53$ & $2.62 \pm 0.70$ & $<0.001^{*}$ & 85.891 & $1.90 \pm 0.65$ & 1.5 & $<0.001^{*}$ & 232.936 \\
\hline $\begin{array}{l}\text { TFLL } \\
\qquad(\mathrm{IPL}+)\end{array}$ & $3.46 \pm 0.54$ & $2.50 \pm 0.95$ & $0.01^{*}$ & 49.171 & $1.60 \pm 0.64$ & 1.9 & $<0.001^{*}$ & 232.148 \\
\hline$P$ value & 0.098 & 0.502 & - & - & $0.021^{*}$ & - & - & - \\
\hline$F$ value & 2.882 & 0.457 & - & - & 5.727 & - & - & - \\
\hline $\begin{array}{l}\text { MGQ } \\
\text { (IPL) }\end{array}$ & $2.20 \pm 0.57$ & $1.86 \pm 0.64$ & $<0.001^{*}$ & 25.242 & $1.48 \pm 0.54$ & 0.7 & $<0.001^{*}$ & 70.248 \\
\hline $\begin{array}{l}\text { MGQ } \\
\quad(\mathrm{IPL}+)\end{array}$ & $2.30 \pm 0.58$ & $1.76 \pm 0.66$ & $<0.001^{*}$ & 35.986 & $1.26 \pm 0.56$ & 1.0 & $<0.001^{*}$ & 110.783 \\
\hline$P$ value & 0.255 & 0.389 & - & - & $0.026^{*}$ & - & - & - \\
\hline$F$ value & 1.324 & 0.754 & - & - & 5.252 & - & - & - \\
\hline $\begin{array}{l}\text { MGEx } \\
\text { (IPL) }\end{array}$ & $2.50 \pm 0.51$ & $1.86 \pm 0.45$ & $<0.001^{*}$ & 87.111 & $1.62 \pm 0.53$ & 0.9 & $<0.001^{*}$ & 98.407 \\
\hline $\begin{array}{l}\text { MGEx } \\
\quad(\mathrm{IPL}+)\end{array}$ & $2.58 \pm 0.50$ & $1.80 \pm 0.45$ & $<0.001^{*}$ & 118.488 & $1.44 \pm 0.50$ & 1.1 & $<0.001^{*}$ & 227.106 \\
\hline$P$ value & 0.209 & 0.444 & - & - & $0.038^{*}$ & - & - & - \\
\hline$F$ value & 1.62 & 0.595 & - & - & 4.567 & - & - & - \\
\hline CFS (IPL) & $4.44 \pm 0.54$ & $4.20 \pm 0.70$ & $<0.001^{*}$ & 15.474 & $4.02 \pm 0.65$ & 0.4 & $<0.001^{*}$ & 35.483 \\
\hline $\begin{array}{l}\text { CFS } \\
\qquad(\mathrm{IPL}+)\end{array}$ & $4.58 \pm 0.70$ & $4.36 \pm 0.60$ & $0.001^{*}$ & 13.821 & $3.96 \pm 0.73$ & 0.6 & $<0.001^{*}$ & 47.613 \\
\hline$P$ value & 0.109 & 0.185 & - & - & 0.652 & - & - & - \\
\hline$F$ value & 2.665 & 1.806 & - & - & 0.206 & - & - & - \\
\hline $\begin{array}{r}\text { LTMH } \\
(\text { IPL })\end{array}$ & $0.146 \pm 0.029$ & $0.170 \pm 0.038$ & $<0.001^{*}$ & 40.552 & $0.186 \pm 0.053$ & 0.04 & $<0.001^{*}$ & 35.254 \\
\hline $\begin{array}{l}\text { LTMH } \\
\qquad(\mathrm{IPL}+)\end{array}$ & $0.147 \pm 0.030$ & $0.180 \pm 0.040$ & $<0.001^{*}$ & 57.266 & $0.204 \pm 0.034$ & 0.06 & $<0.001^{*}$ & 156.713 \\
\hline
\end{tabular}


Table 2 continued

\begin{tabular}{|c|c|c|c|c|c|c|c|c|}
\hline Parameters & Baseline (BL) & $\begin{array}{l}\text { Follow-up } \\
\text { (FU1) }\end{array}$ & $\begin{array}{l}P \text { value } \\
\text { (BL vs. } \\
\text { FU1) }\end{array}$ & $\begin{array}{l}F \text { value } \\
\text { (BL vs. } \\
\text { FU1) }\end{array}$ & $\begin{array}{l}\text { Follow-up } \\
\text { (FU2) }\end{array}$ & $\begin{array}{l}\Delta \\
\text { change } \\
\text { (BL- } \\
\text { FU2) }\end{array}$ & $\begin{array}{l}P \text { value } \\
\text { (BL vs. } \\
\text { FU2) }\end{array}$ & $\begin{array}{l}F \text { value } \\
\text { (BL vs. } \\
\text { FU2) }\end{array}$ \\
\hline$P$ value & 0.389 & $0.022^{*}$ & - & - & $0.003^{*}$ & - & - & - \\
\hline$F$ value & 0.754 & 5.568 & - & - & 9.577 & - & - & - \\
\hline $\begin{array}{l}\text { OSDI } \\
\text { (IPL) }\end{array}$ & $46.34 \pm 6.84$ & $45.55 \pm 5.35$ & 0.89 & 3.019 & $32.54 \pm 6.85$ & 13.8 & $<0.001^{*}$ & 9331.56 \\
\hline $\begin{array}{l}\text { OSDI } \\
\quad(\mathrm{IPL}+)\end{array}$ & $46.36 \pm 6.77$ & $41.92 \pm 5.80$ & $<0.001^{*}$ & 26.65 & $29.76 \pm 4.74$ & 16.6 & $<0.001^{*}$ & 402.337 \\
\hline$P$ value & 0.86 & $<0.001^{*}$ & - & - & $0.001^{*}$ & - & - & - \\
\hline$F$ value & 0.032 & 18.825 & - & - & 11.584 & - & - & - \\
\hline $\begin{array}{l}\text { BCVA } \\
\text { (IPL) }\end{array}$ & $-0.02 \pm 0.01$ & $-0.01 \pm 0.01$ & 0.444 & 0.595 & $-0.02 \pm 0.01$ & 0.00 & 0.159 & 2.042 \\
\hline $\begin{array}{l}\mathrm{BCVA} \\
\quad(\mathrm{IPL}+)\end{array}$ & $-0.02 \pm 0.01$ & $-0.02 \pm 0.01$ & 0.811 & 0.058 & $-0.02 \pm 0.01$ & 0.00 & 0.290 & 1.146 \\
\hline$P$ value & 0.766 & 0.164 & - & - & 0.844 & - & - & - \\
\hline$F$ value & 0.089 & 1.999 & - & - & 0.039 & - & - & - \\
\hline ATU (IPL) & $4.46 \pm 0.84$ & $3.70 \pm 0.76$ & $<0.001^{*}$ & 107.86 & $1.88 \pm 0.63$ & 2.6 & $<0.001^{*}$ & 290.284 \\
\hline $\begin{array}{l}\text { ATU } \\
\quad(\mathrm{IPL}+)\end{array}$ & $4.48 \pm 0.84$ & $3.60 \pm 0.61$ & $<0.001^{*}$ & & $1.72 \pm 0.50$ & 2.8 & $<0.001^{*}$ & 379.95 \\
\hline$P$ value & 0.659 & 0.358 & - & - & 0.159 & - & - & - \\
\hline$F$ value & 0.197 & 0.86 & - & - & 2.042 & - & - & - \\
\hline
\end{tabular}

$B L$ baseline assessment; $F 1$ first follow-up assessment; $F 2$ second follow-up assessment; IPL intense pulsed light; IPL + intense pulsed light combined with heated eye mask; NITBUT non-invasive tear break-up time (seconds); CFS corneal fluorescein staining (score); $M G Q$ meibomian gland quality (scale); MGEx meibomian gland expression (scale); OSDI ocular surface disease index (score); BCVA best-corrected visual acuity ( $\operatorname{LogMAR}) ; A T U$ artificial tear usage (frequency of drops per day)

${ }^{*}$ Statistically significant difference

baseline scores (IPL: $2.62 \pm 0.70, P<0.001$ and $\mathrm{IPL}+: \quad 2.50 \pm 0.95, \quad P=0.01, \quad$ respectively, $P=0.502)$. Mean TFLL scores at the second follow-up were significantly different (IPL: $1.90 \pm 0.65, P<0.001$, vs. IPL $+: 1.60 \pm 0.64$, $P<0.001$, respectively, $P=0.021$ ). Additionally, $\Delta$ change in TFLL was higher in the IPL + group (1.9) compared to the IPL group (1.5) (Table 2).

\section{LTMH}

The mean baseline LTMH measurements for IPL and IPL + groups were $0.146 \pm 0.029$ and $0.147 \pm 0.030$, respectively $(P=0.389)$ (Fig. 5). At the first and second follow-up assessments, significant changes were observed between the groups $(P=0.022$ and $P=0.003$, respectively). 


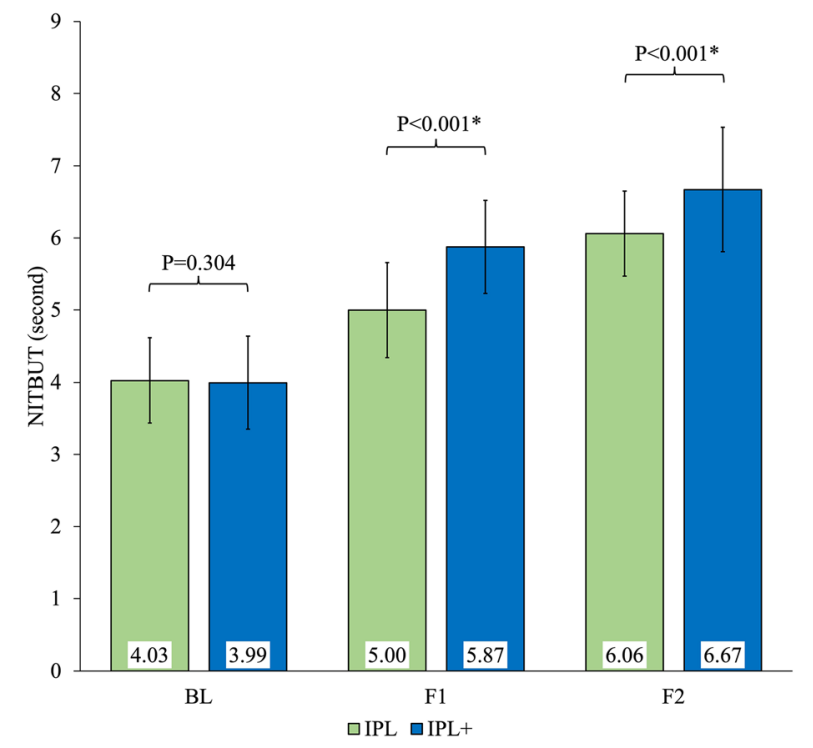

Fig. 3 Comparison of mean NITBUT at different time points between groups

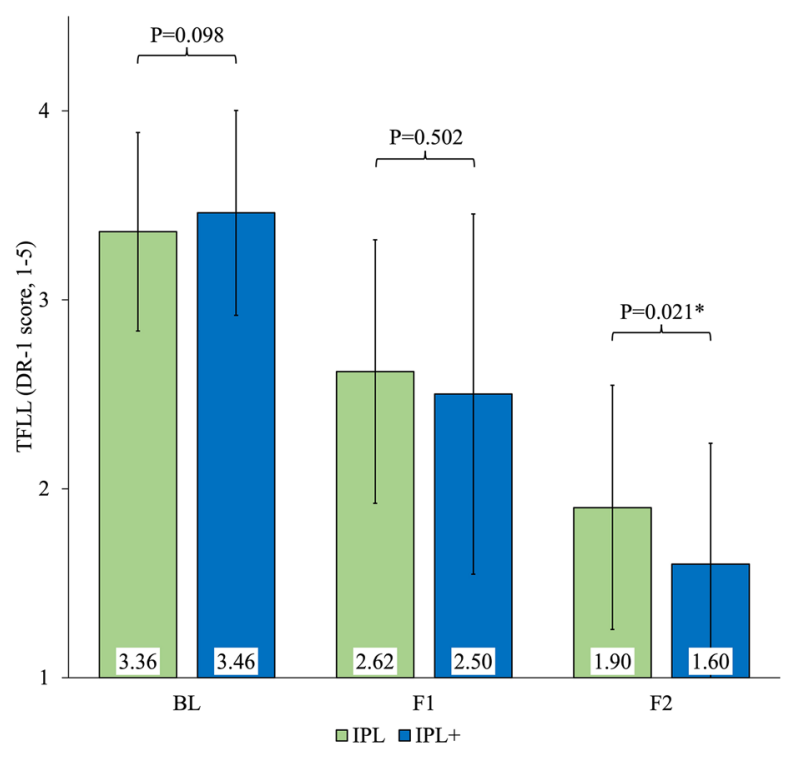

Fig. 4 Comparison of mean TFLL score at different time points between groups

The IPL + group had a higher $\Delta$ change in mean LTMH (IPL: 0.04 vs. IPL +: 0.06) (Table 2).

\section{MGQ and MGEx}

Changes in the MGQ and MGEx of meibomian gland scores are given in Figs. 6 and 7,

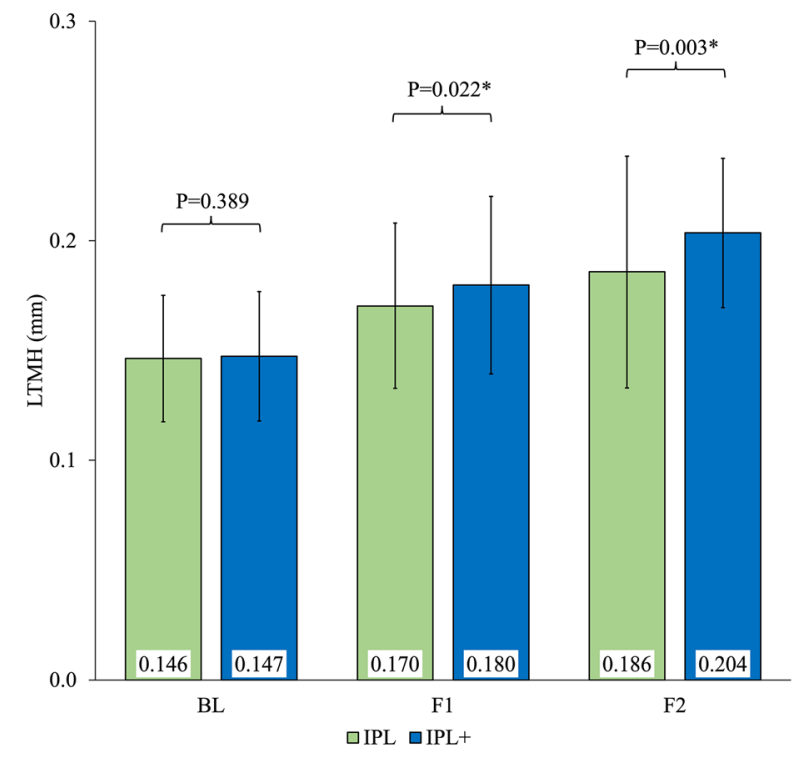

Fig. 5 Comparison of mean LTMH at different time points between groups

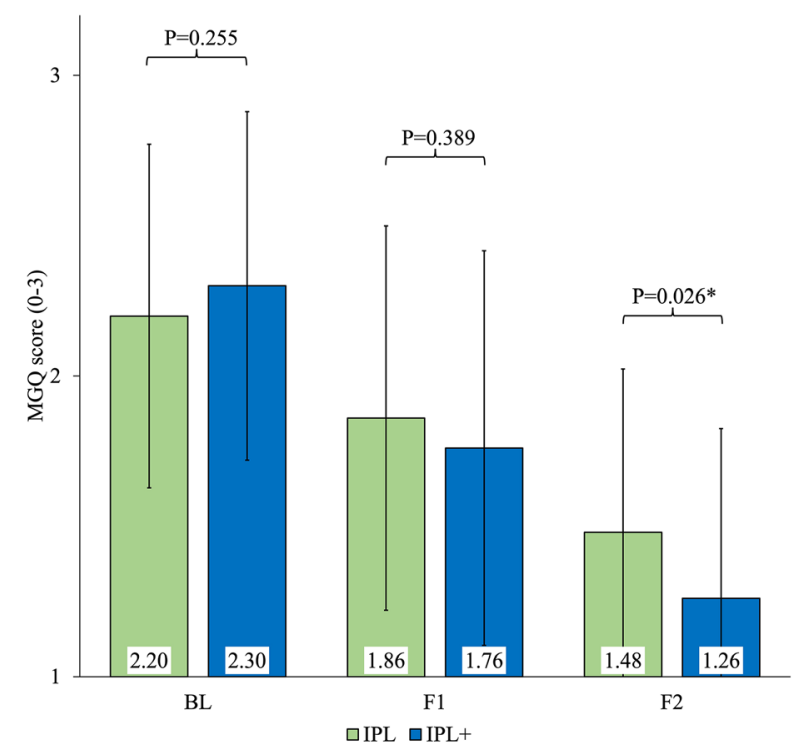

Fig. 6 Comparison of mean MG scores at different time points between groups

respectively. At baseline, mean MGQ score (IPL: $2.20 \pm 0.57$ and IPL $+: 2.30 \pm 0.58, P=0.255$, respectively) and mean MGEx score (IPL: $2.50 \pm 0.51$ and IPL $+: 2.58 \pm 0.50, P=0.209$, respectively) had no statistically significant difference. At the first follow-up assessment, both groups showed significant improvements in 


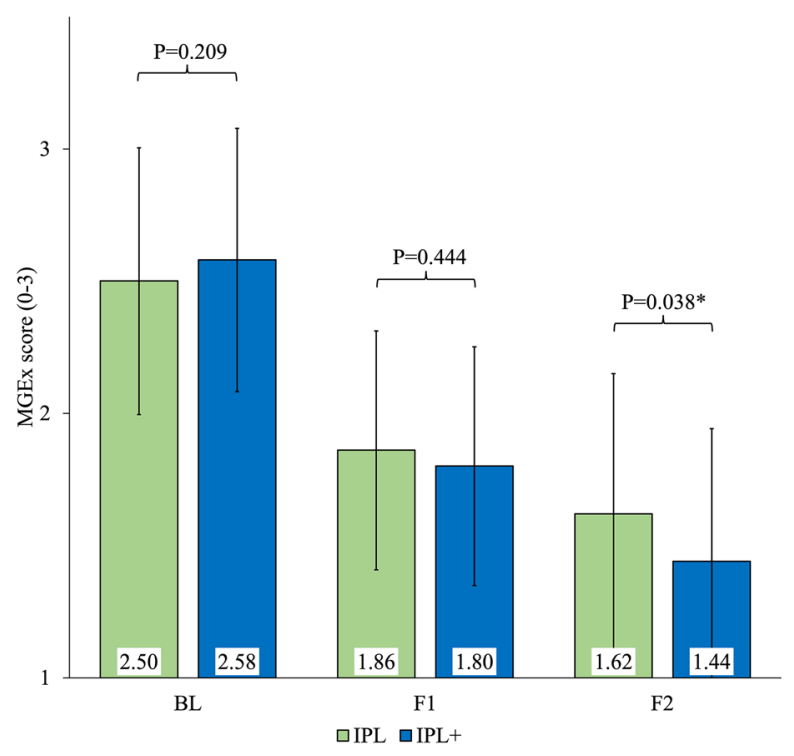

Fig. 7 Comparison of mean MGEx scores at different time points between groups

their MGQ and MGEx scores compared to their respective baseline scores; however, there were no significant differences between the groups (MGQ score: $P=0.389$ and MGEx score: $P=0.444)$. At the final follow-up, significant differences between the groups (MGQ score: $1.48 \pm 0.54, \quad P=0.026$ and MGEx score: $1.62 \pm 0.53, \quad P=0.038, \quad$ respectively) were found. IPL + group was found to have a higher $\Delta$ change in mean MGQ (IPL: 0.7 vs. IPL $+: 1.0$ ) and mean MGEx (IPL: 0.9 vs. IPL +: 1.1 ) scores (Table 2).

\section{CFS}

Mean CFS scores at baseline for IPL and IPL + groups were $4.44 \pm 0.54$ and $4.58 \pm 0.70$, respectively $(P=0.109)$. At the first and second follow-up assessments, no significant changes were observed between the groups $(P=0.185$ and $P=0.652$, respectively) (Table 2 ).

\section{OSDI}

Figure 8 illustrates the mean changes in OSDI scores in IPL and IPL + groups at baseline, first and second follow-ups. Inter-group comparison revealed significant changes at first (IPL:

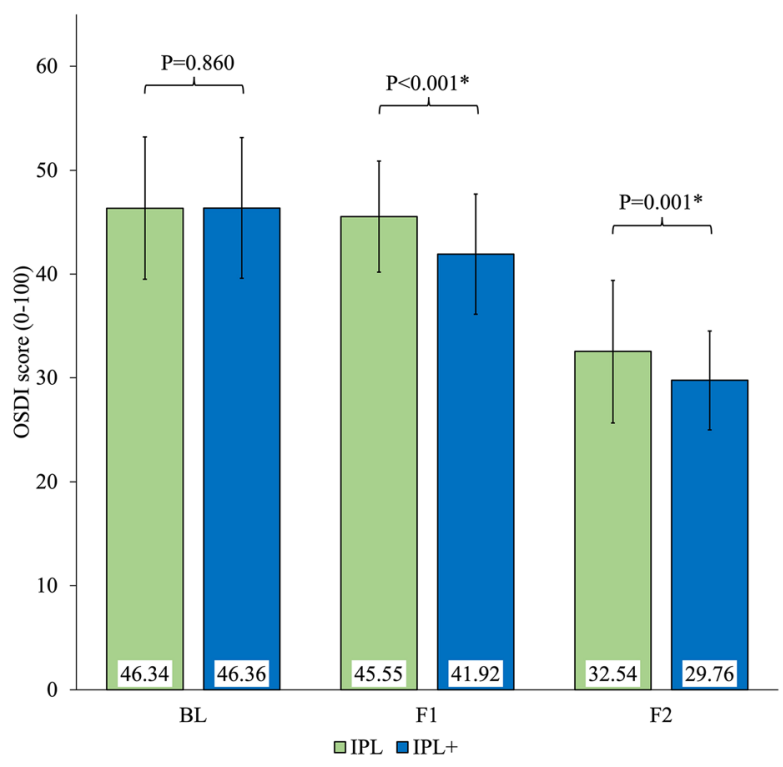

Fig. 8 Comparison of mean OSDI scores at different time points between groups

$45.55 \pm 5.35$ and IPL $+: \quad 41.92 \pm 5.80$, $P<0.001$ ) and second (IPL: $32.54 \pm 6.85$ and IPL $+: \quad 29.76 \pm 4.74, \quad P=0.001)$ follow-ups. IPL + group was found to have a higher $\Delta$ change in mean OSDI score (IPL: 13.8 vs. IPL + : 16.6) (Table 2). Individual mean OSDI scores of participants are represented on a heat map in Fig. 9A, B.

\section{ATU}

Changes in mean ATU of both groups are presented in Table 2. At baseline, mean ATUs in both groups were not significantly different (IPL: $4.46 \pm 0.84$ and IPL $+: 4.48 \pm 0.84$, $P=0.659$ ). Additionally, there were no significant changes between the groups at first (IPL: $3.70 \pm 0.76$ and IPL $+: 3.60 \pm 0.61, P=0.358$ ) and second (IPL: $1.88 \pm 0.63$ and IPL + : $1.72 \pm 0.50, P=0.159)$ follow-ups.

\section{DISCUSSION}

LASIK-associated DE can be frustrating for both patients and clinicians and is the leading complaint following LASIK [40]. Symptoms experienced by post-LASIK DE patients are not 
A

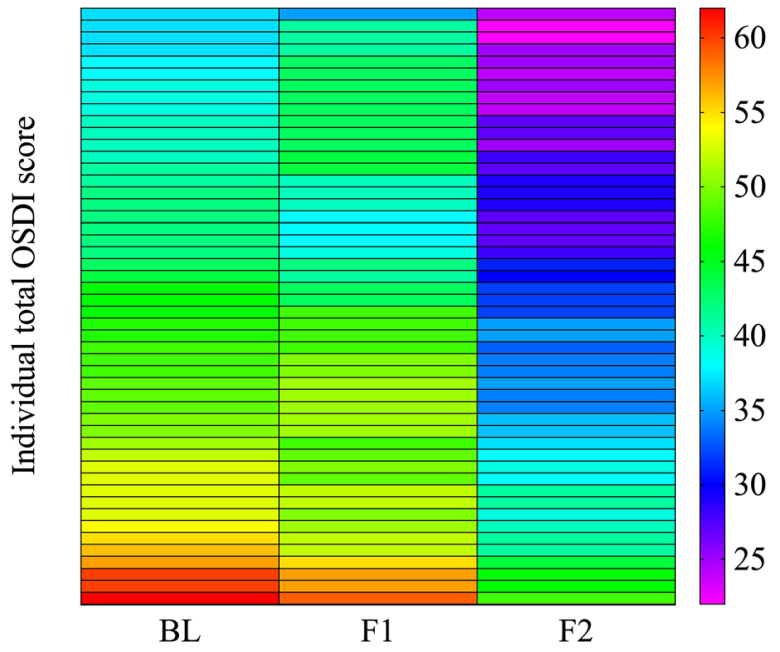

B

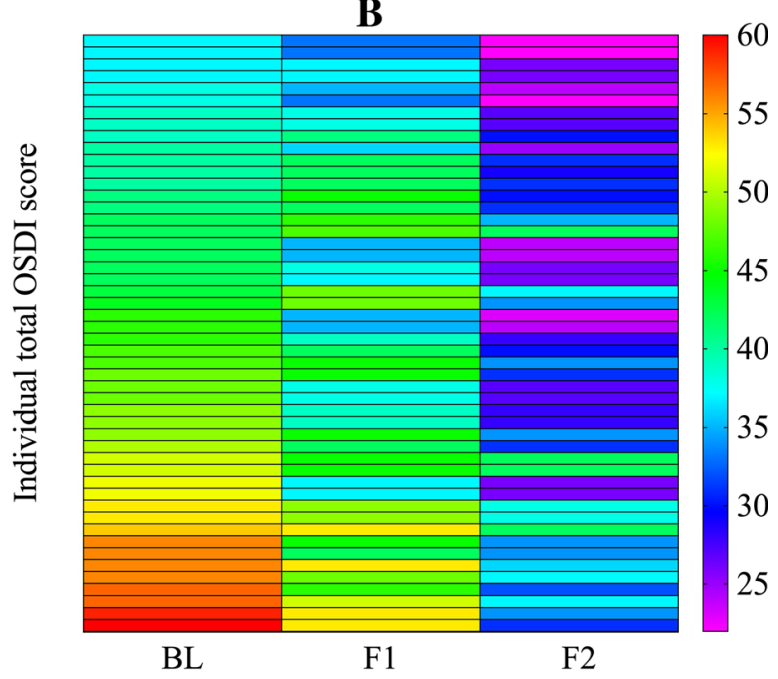

Fig. 9 Heat map depiction of individual OSDI scores. A IPL group, B IPL + group

consistent and comprise a spectrum of pathologies such as neurotrophic disease, injured conjunctival goblet cells, reduced blink rate, tear film instability and aqueous tear deficiency [41]. This results in a combination of pathophysiological factors that causes inflammation of the ocular surface. Several cytokines and chemokines have been documented to increase tears because of evaporative-type MDGdependent DED. DED is a type of ocular surface inflammation where signs and symptoms often do not correlate well, particularly in the mildto-moderate cases. Furthermore, increases in inflammatory cytokine tear concentrations may be responsible for the irritation symptoms and ocular surface disease in DED. This finally results in varying severity in signs and symptoms of DE [11, 42], and tear inflammatory cytokines such as IL-17A, IL-6 and PGE2 have been reported to decrease after IPL treatment [21].

While post-LASIK DE can be managed by standard DE treatment [5], persistent DE symptoms are difficult to treat. Pazo et al. [30] found significant differences between IPL and control groups in objective and subjective DE assessment tests in refractory post-LASIK DE participants.

In the current study, subjects were randomized to IPL (IPL in combination with $0.1 \% \mathrm{SH}$ ) and IPL + (IPL in combination with $0.1 \% \mathrm{SH}$ and HEM). All participants in both groups were treated two times at a 2-week interval; in addition, the IPL + group received HEM daily. Subjective and objective assessments were performed at baseline, 2 weeks from baseline and 4 weeks from baseline. The primary outcome measures were NITBUT, TFLL, LTMH and OSDI. The current study found that participants in both groups had improved signs and symptoms of DED compared to their respective baseline measurements but overall the improvement in the IPL + group was more pronounced. While both the DEWS II [43] committee report and the Japanese DE [31] committee aim to accurately diagnose $\mathrm{DE}$, the current study chose the Japanese DE diagnostic criteria over the DEWS II definition since this study emphasized the diagnostic value such as "visible/clinical changes" whereas the DEWS II report focuses more on "invisible/underlying" causes of DE such as hyperosmolarity, ocular surface inflammation and damage, and neurosensory abnormalities as well as tear film instability [44].

Although mechanisms of post-LASIK DE have been postulated to primarily arise from corneal nerves damage during LASIK surgery. Studies have also concluded that LASIK and other corneal refractive surgeries can significantly reduce proper meibomian gland function [16]. Therefore, improving the TFLL does not 
directly correct the intraoperative injury that contributed to post-LASIK DE; however, improved TFLL enhances the ocular surface environment, which benefits the homeostatic balance of the ocular surface $[27,45]$. As documented by Di Pascuale et al. [27], improving the TFLL in post-LASIK DE participants with the aid of an eyelid warming device improved tear film stability. Furthermore, Di Pascuale et al. documented a high prevalence of tear film lipid deficiency in subjects with persistent dry eye after LASIK. Preoperative risk avoidance [40] and intraoperative strategies such as hinge positioning have been explored to lessen the damage to corneal nerves during LASIK $[46,47]$.

The effectiveness of HY in treating DED has been tied to various mechanisms of action. In vitro studies suggest that HY inactivates the CD44 adhesion molecule, a receptor of HY found to be overexpressed on the ocular surface of subjects with DED [48]. HY has been suggested to have a localized anti-inflammatory effect. The viscous nature of HY can act as a lubricant and reduce friction when the eye blinks. Additionally, water-retentive properties of $\mathrm{HY}$ have been reported to be 1000-fold its own weight, thereby hydrating the ocular surface and reducing evaporation of tears $[16,45]$.

IPL treatment in combination with HEM improves meibomian gland function and meibum secretion, which are vital functions that stabilize the tear film and promotes ocular surface integrity $[16,45]$. As suggested by prior research on meibum secretion, the HEM used in our study reached $>40-\mathrm{C}$ (Fig. 2) and therefore was adequate to mobilize the flow of meibum into the tear film [46, 47]. Fortifying refractory DE subjects tear film with a polar and nonpolar lipid base, ofloxacin eye ointment improved signs and symptoms of DE [48]. Improved secretion of TFLL offers a physical protective boundary and in tandem with blinking facilitates its distribution on the ocular surface [49]. According to Foulks et al. [50], severity of DE symptoms appears to correlate to lipid layer thickness. Therefore, DE treatments that replenish or enhance meibum production have been found to improve signs and symptoms of DED [51, 52]. In addition, lipids expressed by the meibomian gland have been documented to be antimicrobial, impair gram-positive and gram-negative bacterial growth and further fortify the ocular surface [53]. The active control (IPL group) in this study was standardized as two IPL sessions, 2 weeks apart. The study results (Table 2) indicate that after 4 weeks, the IPL + group showed significantly better improvement of meibomian gland function and reduction of DE signs and symptoms than IPL treatment alone. This significant difference between IPL and IPL + in these outcomes was documented after controlling for participant demographic and baseline characteristics .

The limitations of the study are as follows: The participants undergoing treatment (IPL and IPL +) and the investigators were not masked. Although the dosage (frequency of drops) of $0.1 \% \mathrm{SH}$ used by participants at baseline was similar $(P=0.659)$ between the groups, the dosage changed during the study as participants were less reliant and reduced the frequency of SH usage. LASIK surgery preoperative and immediately postoperative and ocular surface data of the participants in this study were not available since participants had undergone refractive surgeries in various other centers. The lack of a large sample size also limits this study because persistent post-LASIK DE is not common since most post-LASIK DEs are transient [3]. Finally, managing post-LASIK DE with IPL is a relatively new concept, and optimizing the dose and duration will be explored in future studies .

\section{CONCLUSION}

In summary, the results of this study suggest that post-LASIK DE signs and symptoms can be improved by combining therapies such as IPL, $0.1 \%$ SH and HEM. Increased TFLL due to a combination of $0.1 \% \mathrm{SH}$ and HEM seems to have a greater positive impact on the subjective and objective DE measurements in participants with persistent post-LASIK DE. 


\section{ACKNOWLEDGEMENTS}

We thank the participants in this study. This work was supported by He Eye Specialist Hospital, Shenyang, China. The authors have no proprietary interest in any of the products mentioned in this article.

Funding. This study was entirely funded by He Eye Specialist Hospital, Shenyang, China. No support was received for the publication of this article. The journal's Rapid Service Fees was funded by the authors.

Authorship. All named authors meet the International Committee of Medical Journal Editors (ICMJE) criteria for authorship for this article, take responsibility for the integrity of the work as a whole, and have given their approval for this version to be published.

Author Contributions. Conceptualization: $\mathrm{Yi} \mathrm{Wu}$, Ling $\mathrm{Xu}$, and Emmanuel Pazo, Methodology: Yi Wu, Ling Xu, Yilin Song, Qing Zhang, Guanghao Qin, Lanting Yang, Jinfei Ma, Christoph Palme, Jonathan E Moore, Emmanuel Eric Pazo, Wei He. Formal analysis and investigation: Yi $\mathrm{Wu}$ and Emmanuel Pazo. Writingoriginal draft preparation: Yi Wu. Writing-review and editing: $\mathrm{Yi} \mathrm{Wu}$, Ling $\mathrm{Xu}$, and Emmanuel Pazo. Methodology: $\mathrm{Yi} \mathrm{Wu}$, Ling $\mathrm{Xu}$, Emmanuel Eric Pazo, and Wei He. Funding acquisition: Ling $\mathrm{Xu}$, and Wei He. Resources: Ling $\mathrm{Xu}$, Wei $\mathrm{He}$, Supervision: Ling $\mathrm{Xu}$, Emmanuel Pazo and Wei He.

Disclosures. The authors: $\mathrm{Yi} \mathrm{Wu}$, Ling $\mathrm{Xu}$, Yilin Song, Qing Zhang, Guanghao Qin, Lanting Yang, Jinfei Ma, Christoph Palme, Jonathan E Moore, and Emmanuel Pazo, and Wei He confirm that they have nothing to disclose.

Compliance with Ethics Guidelines. The study was conducted according to the guidelines of the Declaration of Helsinki and approved by the Institutional Review Board of the He Eye Specialist Hospital, Shenyang, China (IRB2019K002.01), approved on 23 January 2019. Written informed consent was obtained from the patients for publication of this case report and any accompanying images. This manuscript includes no identifiable patient information.

Data Availability. The data used to support the findings of this study are available from the corresponding author upon request.

Open Access. This article is distributed under the terms of the Creative Commons Attribution-Non-Commercial 4.0 International License (http://creativecommons.org/licenses/ by-nc/4.0/), which permits any noncommercial use, distribution, and reproduction in any medium, provided you give appropriate credit to the original author(s) and the source, provide a link to the Creative Commons license, and indicate if changes were made.

Rights and Permissions. This article is distributed under the terms of the Creative Commons Attribution-Non Commercial 4.0 International License (http://creativecommons. org/licenses/ by-nc/4.0/), which permits any noncommercial use, distribution, and reproduction in any medium, provided you give appropriate credit to the original author(s) and the source, provide a link to the Creative Commons license, and indicate if changes were made.

Open Access. This article is licensed under a Creative Commons Attribution-NonCommercial 4.0 International License, which permits any non-commercial use, sharing, adaptation, distribution and reproduction in any medium or format, as long as you give appropriate credit to the original author(s) and the source, provide a link to the Creative Commons licence, and indicate if changes were made. The images or other third party material in this article are included in the article's Creative Commons licence, unless indicated otherwise in a credit line to the material. If material is not included in the article's Creative Commons licence and your intended use is not permitted by statutory regulation or exceeds the permitted use, you will need to obtain permission directly from the copyright holder. To view a copy of this licence, 
visit http://creativecommons.org/licenses/bync/4.0/.

\section{REFERENCES}

1. Murakami Y, Manche EE. Prospective, randomized comparison of self-reported postoperative dry eye and visual fluctuation in LASIK and photorefractive keratectomy. Ophthalmology. 2012;119:2220-4. https://doi.org/10.1016/j.ophtha.2012.06.013.

2. Wang B, Naidu RK, Chu R, Dai J, Qu X, Zhou H. Dry eye disease following refractive surgery: a 12-month follow-up of SMILE versus FS-LASIK in high myopia. J Ophthalmol. 2015;2015:1-8. https://doi.org/10. $1155 / 2015 / 132417$.

3. Sambhi R-DS, Sambhi GDS, Mather R, MalvankarMehta MS. Dry eye after refractive surgery: a metaanalysis. Can J Ophthalmol. 2020;55:99-106. https://doi.org/10.1016/j.jcjo.2019.07.005.

4. Craig JP, Nichols KK, Akpek EK, Caffery B, Dua HS, Joo C-K, et al. TFOS DEWS II definition and classification report. Ocul Surf. 2017;15:276-83. https:// doi.org/10.1016/j.jtos.2017.05.008.

5. Rosenfeld SI. Evaluation and management of PostLASIK dry eye syndrome. Int Ophthalmol Clin. 2010;50:191-9. 0b013e3181e2469b.

6. Toda I. Dry eye after LASIK. Invest Ophthalmol Vis Sci. 2018;59:DES109. https://doi.org/10.1167/iovs. 17-23538.

7. Nettune GR, Pflugfelder SC. Post-LASIK tear dysfunction and dysesthesia. Ocul Surf. 2010;8:135-45. https://doi.org/10.1016/S1542-0124(12)70224-0.

8. Rodriguez AE, Alio JL, Rodriguez-Prats JL. Decrease in goblet cell density following lasik as a cause of dry eye post surgery. Invest Ophthalmol Vis Sci. 2006;47:273-273.

9. Shin S, research YL-O, 2006 undefined. Conjunctival changes induced by LASIK suction ring in a rabbit model. karger.com [Internet]; Available from: https://www.karger.com/Article/Abstract/96229. Accessed 21 Aug 2021.

10. Zhang Q, Wu Y, Song Y, Qin G, Yang L, Talwar SS, et al. Screening evaporative dry eyes severity using an infrared image. J Ophthalmol. 2021;2021:1-8. https://doi.org/10.1155/2021/8396503.

11. Chan T, Ye C, Chan KP. Evaluation of point-of-care test for elevated tear matrix metalloproteinase 9 in
post-LASIK dry eyes. Br J Ophthalmol. 2016;100: 1188-91. https://doi.org/10.1136/bjophthalmol2015-307607.

12. González-Pérez J, Villa-Collar C, González-Méijome JM, Porta NG, Parafita MÁ. Long-term changes in corneal structure and tear inflammatory mediators after orthokeratology and LASIK. Invest Opthalmol Vis Sci. 2012;53:5301. https://doi.org/10.1167/iovs. 11-9155.

13. Lee J, Ryu C, Kim J, Kim E, Refractive HK-J of C\&, 2000 undefined. Comparison of tear secretion and tear film instability after photorefractive keratectomy and laser in situ keratomileusis. Elsevier [Internet]; Available from: https://www.sciencedirect. com/science/article/pii/S0886335000005666. Accessed 21 Aug 2021.

14. Benitez-del-Castillo J, Rio T del, Cornea TI-, 2001 undefined. Decrease in tear secretion and corneal sensitivity after laser in situ keratomileusis. journals.lww.com [Internet]; Available from: https:// journals.lww.com/corneajrnl/fulltext/2001/01000/ decrease_in_tear_secretion_and_corneal_ sensitivity.5.aspx. Accessed 21 Aug $202 \overline{1}$.

15. Wan $\mathrm{T}$, Jin $\mathrm{X}$, Lin $\mathrm{L}, \mathrm{Xu} \mathrm{Y}$, Zhao Y. Incomplete blinking may attribute to the development of meibomian gland dysfunction. Curr Eye Res. 2016;41: 179-85. https://doi.org/10.3109/02713683.2015. 1007211.

16. Jung JW, Kim JY, Chin HS, Suh YJ, Kim T, Seo KY. Assessment of meibomian glands and tear film in post-refractive surgery patients. Clin Exp Ophthalmol. 2017;45:857-66. https://doi.org/10.1111/ceo. 12993.

17. Ang B, Sng J, Wang P, Htoon H, reports LT-S, 2017 undefined. Sodium hyaluronate in the treatment of dry eye syndrome: a systematic review and metaanalysis. nature.com [Internet]; Available from: https://www.nature.com/articles/s41598-01708534-5. Accessed 14 Oct 2021.

18. Raulin C, Greve B. Medicine HG-L in S and $2003 \mathrm{U}$ IPL technology: a review. Wiley Online Library; 2003.

19. Song Y, Yu S, He X, Yang L, Wu Y, Qin G, et al. Tear film interferometry assessment after intense pulsed light in dry eye disease: A randomized, single masked, sham-controlled study. Contact Lens and Anterior Eye [Internet]. Elsevier; 2021;101499. Available from: https://www.sciencedirect.com/ science/article/pii/S1367048421001284. Accessed 13 Sep 2021.

20. Heymann WR. Intense pulsed light. J Am Acad Dermatol. 2007; 56:466-7. 
21. Liu R, Rong B, Tu P, Tang Y, Song W, Toyos R, et al. Analysis of cytokine levels in tears and clinical correlations after intense pulsed light treating meibomian gland dysfunction. Am J Ophthalmol. 2017;183:81-90. https://doi.org/10.1016/j.ajo. 2017.08.021.

22. Bäumler W, Vural E, Landthaler M, Muzzi F, Shafirstein G. The effects of intense pulsed light (IPL) on blood vessels investigated by mathematical modeling. Lasers Surg Med. 2007;39:132-9.

23. Cao Y, Huo R, Feng Y, Li Q, Wang F. Effects of intense pulsed light on the biological properties and ultrastructure of skin dermal fibroblasts: potential roles in photoaging. Photomed Laser Surg. 2011;29:327-32.

24. Jeng S, Chen J, Chang L, Chen C, Shih H, Chou T, et al. Beneficial effect of intense pulsed light on the wound healing in diabetic rats. Lasers Surg Med. 2020;52:530-6.

25. Pazo EE, Huang H, Fan Q, Zhang C, Yue Y, Yang L, et al. Intense pulse light for treating post-LASIK refractory dry eye. Photobiomodul Photomed Laser Surg. 2020. https://doi.org/10.1089/photob.2020. 4931.

26. Chen Y, Li J, Wu Y, Lin X, Deng X, Yun-e Z. Comparative evaluation in intense pulsed light therapy combined with or without meibomian gland expression for the treatment of meibomian gland dysfunction. Curr Eye Res. 2021;46:1125-31.

27. di Pascuale MA, Liu T-S, Trattler W, Tseng SCG. Lipid tear deficiency in persistent dry eye after laser in situ keratomileusis and treatment results of new eye-warming device. J Cataract Refract Surg. 2005;31:1741-9. https://doi.org/10.1016/j.jcrs. 2005.02.041.

28. Johnson ME, Murphy PJ, Boulton M. Effectiveness of sodium hyaluronate eyedrops in the treatment of dry eye. Graefe's Arch Clin Exp Ophthalmol. 2006;244:109-12. https://doi.org/10.1007/s00417005-0028-1.

29. Williamson JF, Huynh K, Weaver MA, Davis RM. Perceptions of dry eye disease management in current clinical practice. Eye Contact Lens. 2014;40: 111-5. 0000000000000020 .

30. Pazo EE, Huang H, Fan Q, Zhang C, Yue Y, Yang L, et al. Intense pulse light for treating post-LASIK refractory dry eye. Photobiomodul Photomed Laser Surg. 2021;39:155-63. https://doi.org/10.1089/ photob.2020.4931.

31. Uchino Y, Uchino M, Dogru M, Ward S, Yokoi N, Tsubota K. Changes in dry eye diagnostic status following implementation of revised Japanese dry eye diagnostic criteria. Jpn J Ophthalmol. 2011;56: 8-13. https://doi.org/10.1007/s10384-011-0099-y.

32. Zhang X, Yang L, Zhang Q, Fan Q, Zhang C, You Y, et al. Reliability of Chinese Web-based Ocular Surface Disease Index (C-OSDI) Questionnaire in Dry Eye Patients: A Randomized, Crossover Study. International journal of ophthalmology. 2021;in press.

33. Xie W, Jiang L, Zhang X, Xu Y, Yao Y. Eyelid margin cleaning using Deep Cleaning Device for the treatment of meibomian gland dysfunction-associated dry eye: a preliminary investigation. J Zhejiang UnivSci B. 2019;20:679-86. https://doi.org/10. 1631/jzus.B1900091.

34. Best N, Drury L, Wolffsohn JS. Clinical evaluation of the Oculus Keratograph. Contact Lens Anterior Eye. 2012;35:171-4. https://doi.org/10.1016/j.clae. 2012.04.002.

35. Hosaka E, Kawamorita T, Ogasawara Y, Nakayama $\mathrm{N}$, Uozato $\mathrm{H}$, Shimizu K, et al. Interferometry in the evaluation of precorneal tear film thickness in dry eye. Am J Ophthalmol. 2011;151:18-23.e1.

36. Yang L, Pazo EE, Zhang Q, Wu Y, Song Y, Qin G, et al. Treatment of contact lens related dry eye with intense pulsed light. Contact Lens Anterior Eye. 2021. https://doi.org/10.1016/j.clae.2021.101449.

37. Zhang X-M, Zhang Q, Fan Q-X, Zhang C, You Y, Zhang C-G, et al. Reliability of Chinese web-based ocular surface disease index questionnaire in dry eye patients: a randomized, crossover study. Int J Ophthalmol. 2021;14:834-43.

38. Hulley SB. Designing clinical research [Internet]. Lippincott Williams \& Wilkins; 2007. Available from: https://books.google.com.hk/books?hl= en\&lr=\&id=_7UWxJ5erSsC\&oi=fnd $\&$ pg $=$ PR13\&dq= Designing+clinical+research+:

+ an+epidemiologic + approach.\&ots $=$ YPPxeOu1tN\&sig= 2yr1KI1oIFslp49YOiO68PcNpM8\&redir_esc=y\&hl= zh-CN\&sourceid=cndr\#v=onepage\&q=Designing $\%$ 20clinical\%20research\%20\%3A\%20an\%

20epidemiologic\%20approach.\&f=false. Accessed 26 Jul 2021.

39. Chow S, Shao J, Wang H, Lokhnygina Y. Sample size calculations in clinical research [Internet]. 2017. Available from: https://api.taylorfrancis.com/ content/books/mono/download?identifierName= doi\&identifierValue $=10.1201 /$

9781315183084\&type=googlepdf. Accessed $26 \mathrm{Jul}$ 2021.

40. Cohen E, Spierer O. Dry eye post-laser-assisted in situ keratomileusis: major review and latest 
updates. J Ophthalmol. 2018;2018:1-9. https://doi. org/10.1155/2018/4903831.

41. Shtein RM. Post-LASIK dry eye. Expert Rev Ophthalmol. 2011;6:575-82. https://doi.org/10.1586/ eop.11.56.

42. Roda M, Corazza I, Bacchi Reggiani ML, Pellegrini M, Taroni L, Giannaccare G, et al. Dry eye disease and tear cytokine levels - a meta-analysis. Int J Mol Sci. 2020;21:3111. https://doi.org/10.3390/ ijms21093111.

43. Wolffsohn JS, Arita R, Chalmers R, Djalilian A, Dogru M, Dumbleton K, et al. TFOS DEWS II diagnostic methodology report. Ocul Surf. 2017;15: 539-74. https://doi.org/10.1016/j.jtos.2017.05.001.

44. Shimazaki J. Definition and diagnostic criteria of dry eye disease: historical overview and future directions. Invest Ophthalmol Vis Sci. 2018;59: DES7. https://doi.org/10.1167/iovs.17-23475.

45. Fan Q, Pazo EE, You Y, Zhang CC, Zhang CC, Xu L, et al. Subjective quality of vision in evaporative dry eye patients after intense pulsed light. Photobiomodul Photomed Laser Surg. 2020. https://doi. org/10.1089/photob.2019.4788.

46. Donnenfeld ED, Solomon K, Perry HD, Doshi SJ, Ehrenhaus M, Solomon R, et al. The effect of hinge position on corneal sensation and dry eye after LASIK. Ophthalmology. 2003;110:1023-9. https:// doi.org/10.1016/S0161-6420(03)00100-3.

47. Mian S, Li A, Dutta S, ... DM-J of C\&, 2009 undefined. Dry eyes and corneal sensation after laser in situ keratomileusis with femtosecond laser flap creation: effect of hinge position, hinge angle, and flap thickness. Elsevier [Internet]; Available from: https://www.sciencedirect.com/science/article/pii/ S0886335009008220. Accessed 2 Sep 2021.

48. Nelson J, ophthalmology VH-A of, 1983 undefined. Cellulose acetate impressions of the ocular surface: dry eye states. jamanetwork.com [Internet]; Available from: https://jamanetwork.com/journals/ jamaophthalmology/article-abstract/634837. Accessed 14 Oct 2021.

49. Nakamura M, Hikida M, Nakano T, Ito S, Cornea TH-, 1993 undefined. Characterization of water retentive properties of hyaluronan. europepmc.org
[Internet]; Available from: https://europepmc.org/ article/med/8306665. Accessed 14 Oct 2021.

50. Hamano T, Horimoto K, ... ML-J journal of, 1996 undefined. Sodium hyaluronate eyedrops enhance tear film stability. europepmc.org [Internet]; Available from: https://europepmc.org/article/med/ 8739501. Accessed 14 Oct 2021.

51. Yang L, Pazo EE, Qin G, Zhang Q, Wu Y, Song Y, et al. Effect of intense pulsed light on anterior corneal aberrations and quality of vision in patients with evaporative dry eye. Photobiomodul Photomed Laser Surg. 2021;39:185-95. https://doi.org/ 10.1089/photob.2020.4953.

52. Toyos R, McGill W, Briscoe D. Intense pulsed light treatment for dry eye disease due to meibomian gland dysfunction; a 3-year retrospective study. Photomed Laser Surg. 2015;33:41-6. https://doi. org/10.1089/pho.2014.3819.

53. Borchman D, Foulks GN, Yappert MC, Bell J, Wells E, Neravetla S, et al. Human meibum lipid conformation and thermodynamic changes with meibomian-gland dysfunction. Invest Opthalmol Vis Sci. 2011;52:3805. https://doi.org/10.1167/iovs.106514.

54. Goto E, Dogru M, Fukagawa K, Uchino M, Matsumoto Y, Saiki M, et al. Successful tear lipid layer treatment for refractory dry eye in office workers by low-dose lipid application on the full-length eyelid margin. Am J Ophthalmol. 2006;142:264-270.e1. https://doi.org/10.1016/j.ajo.2006.03.022.

55. Foulks GN. The correlation between the tear film lipid layer and dry eye disease. Surv Ophthalmol. 2007;52:369-74. https://doi.org/10.1016/j. survophthal.2007.04.009.

56. Wang MTM, Cho I, Jung SH, Craig JP. Effect of lipid-based dry eye supplements on the tear film in wearers of eye cosmetics. Contact Lens Anterior Eye. 2017;40:236-41. https://doi.org/10.1016/j. clae.2017.03.001.

57. Fukuoka S, Arita R. Tear film lipid layer increase after diquafosol instillation in dry eye patients with meibomian gland dysfunction: a randomized clinical study. Sci Rep. 2019;9:9091. https://doi.org/10. 1038/s41598-019-45475-7. 